\title{
Headache diagnosis in an urgency and emergency unit: Public Health Relevance and its relationship with cost
}

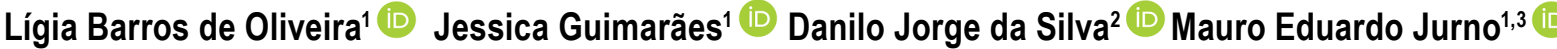

${ }^{1}$ Hospital Foundation of the State of Minas Gerais - FHEMIG, Barbacena, Brazil

2 University Hospital, Federal University of Juiz de Fora, Brazil

${ }^{3}$ Barbacena School of Medicine, Barbacena, Brazil

$\triangle$

Lígia Barros de Oliveira, R João Pinheiro, 923, ap 502, 36201. 128, Barbacena-MG, Brazil. ligiabarrosdeoliveira@yahoo. com.br

Edited by:

Marcelo Moraes Valença
Keywords:

Migraine

Diagnosis

Headache

Health Expenditures

Emergency Medical Services

Public Health

\begin{abstract}
Background

Headache is a common symptom that affects a significant portion of the general population. It constitutes a challenge for diagnosis in urgency and emergency care services, due to headache's clinical variability and diverse possible etiologies, besides the limited time and resources of these facilities. Because of this insufficiency and the potential severity associated with the condition, headaches generate considerable expenditures to health systems, related to both diagnostic discrimination and treatment.

Objective

Evaluating the diagnostic resources used on headache patients care, as well as its Public Health Relevance and relation to cost in an Emergency and Urgency Care unit.

Methods

Cross-section study analyzing 450 medical records of patients with headache complaints in the time frame from January 1, 2019, and December 31, 2019. Patients were categorized according to the type of headache (primary and secondary), specialized evaluation, complementary exams used in the diagnosis, hospital observation time, and the final expenditure in each patient's care.

Results

The total estimated expenditures related to headache care equaled US $\$ 90,855.60$ (average US $\$ 201.90$ per patient). $38.9 \%$ of cases corresponded to primary headaches and $31.1 \%$ to secondary headaches. $30 \%$ of cases could not be classified. The resources utilized for secondary headaches diagnosis differed significantly from those used in primary headache diagnosis. However, the final expenditures were similar to both groups.

\section{Conclusion}

The socio-economic impact caused by headaches is unquestionable. It is a highly frequent symptom and both its etiological distinction and adequate treatment require solid evaluation. Due to the resources spent in its evaluation and monitoring, headaches can be considered a public health problem. Therefore, this study suggests that resources should be allocated in the health education and professional training for the proper conduction of these patients, so that they may benefit from an optimized treatment of their condition without overwhelming the health system.
\end{abstract}




\section{Introduction}

eadache is a universally occurring symptom that affects a significant portion of the general population. In Brazil, the estimated annual headache prevalence is $61.6 \%$ among men and $77.8 \%$ among women. ${ }^{1}$ It is also a frequent occurrence in Emergency Care Units, prompting a high number of consultations.

In Urgency and Emergency Care Units, non-traumatic headache complaints represent from $0.5 \%$ to $2.8 \%$ of medical care. Despite the apparent low frequency, headaches pose a considerable challenge to these facilities, due both to the high variability of their clinical presentation and the wide range of possible diagnoses, which range from benign conditions to high morbimortality causes, and the time and resources available for proper patient assessment. $^{2}$

A Canadian study conducted in the emergency department of a tertiary hospital showed that in $37.5 \%$ of neurological cases, a consensus was not reached between the initial emergency care diagnosis and the eventual final diagnosis given by a neurologist. Among these neurological conditions, primary headaches presented one of the highest dissent rates. ${ }^{3}$ A systematic review carried out in 2014 concluded that only $56 \%$ of migraine patients, a prevalent type of primary headache, received the correct diagnosis when they sought urgency and emergency services. ${ }^{4}$

The principal function of an emergency physician when assessing a patient with headache complaints is to identify life-threatening causes and promptly treating them. Moreover, the physician should provide safe and efficacious treatment for the pain. ${ }^{2}$ In order to do so, it is recommended to differentiate primary headaches (recurrent headache crises are the main symptom) from secondary headaches (the headache is a symptom of an underlying systemic or neurological disease)..$^{5}$ The etiological definition of the secondary headache generally requires a complementary test, which is not the case for primary headaches. ${ }^{5,6}$ In this scenario, it is expected that patients presenting secondary headache clinical features represent a higher expenditure of human and material resources for the health system.

However, primary headaches generate high costs to the health system. They are also one of the main causes of incapacity in the world. The Brazilian public health system estimated annual expenditures with migraine care equaled US\$ 140 million. ${ }^{7}$ The evaluated indirect costs related to migraine-related absenteeism and presenteeism equaled US $\$ 18.6$ billion per year. ${ }^{8}$

The Italian National Health System's (NHS) average annual cost related to the handling of patients diagnosed with episodic migraine or chronic migraine at a tertiary referral center for headache, in terms of hospitalizations equaled $€ 28$ per patient. ${ }^{\text {? }}$
This paper aimed at evaluating the diagnosis, the utilized resources, its Public Health Relevance and the associated costs in the care of patients with headache complaints in the Emergency and Urgency Unit of the Dr. José Américo Barbacena City Regional Hospital, which belongs to the FHEMIG network.

\section{Methods}

A transversal retrospective cohort study was conducted through the analysis of the medical records of patients treated with headache complaints who were admitted to the emergency department of the Regional Hospital of Barbacena, Minas Gerais, from January 1, 2019, to December 31, 2019.

\section{Patients Selection}

The study included all consultations conducted in the Emergency and Urgency Unit of Barbacena City's Dr. José Américo Regional Hospital with an initial headache diagnosis whose registration in the electronic records included the following International Classification of Diseases (ICD-10). ${ }^{10}$

\section{R51: Headache}

G43: Migraine

G43.0: Migraine without aura (common migraine)

G43.1: Migraine with aura (classic migraine)

G43.3: Complicated Migraine

G43.8: Other migraine

G43.9: Migraine, unspecified

G44: Other headache syndromes

G44.0: Cluster headaches

G44.1: Vascular headache, not elsewhere classified

G44.2: Tension-type headache

G44.3: Chronic post-traumatic headache

G44.4: Drug-induced headache, not elsewhere classified

G44.8: Other specified headache syndromes

The study's exclusion criteria were: patients under 18 years of age and consultations in which headache was not part of the patient's initial complaints.

\section{Evaluated Variables}

The selected patients were evaluated according to the following: age; sex; initial headache diagnosis according to the ICD-10; specialized evaluation (neurologist); lumbar puncture and CSF analysis realization; head computed tomography $(\mathrm{CT})$; registered final diagnosis; total cost of consultation, discriminating imaging test costs and medication costs (based on documentation provided 
by the hospital's billing department); hospitalization and hospital observation time.

After medical records were analyzed, patients were reclassified according to their final diagnosis as either primary headache or secondary headache, following the criteria provided by the International Classification of Headache Disorders (ICHD-3). ${ }^{5}$

Afterward, the aforementioned variables were analyzed according to the primary headache and secondary headache diagnoses.

A comparison was drawn between the number of consultations selected for this study (headache patients) and the total number of consultations with patients above 18 years of age at the Barbacena City Regional Hospital in 2019.

\section{Data Analysis}

The data collected through the analysis of medical records were transcribed into an electronic spreadsheet and processed in the statistical software SPSS Statistics 22.0 .

Relative and absolute distributions were calculated for qualitative variables. Continuous variables were verified under the Kolmogorov-Smirnov method normality percepts and listed as the measure for central tendency and mean deviation, and standard deviation for parametric or median distribution, and interquartile distance for non-parametric distributions.

The existence of a relationship between variables was measured through chi-square tests and Fisher's exact test. The existence of a relationship between qualitative and quantitative variables was measured through Student's $t$-test, ANOVA, Mann-Whitney, or Kruskal-Wallis $U$ tests, as indicated. The study considered as statistically significant differences those with $p$ value was under 0.05

\section{Results}

A total of 460 patient medical records were analyzed, which corresponded to 501 consultations due to headache complaints in 2019. Sixteen consultations in which headache was not part of the initial complaints were excluded. 35 consultations for under 18 years of age patients were also removed. Thus, 450 consultations were included in the analysis (Figure 1).

The total number of consultations at Barbacena City Regional Hospital emergency care in 2019 for above 18 years of age patients equaled 24,809 . Thus $1.8 \%$ of hospital visits were motivated by headache complaints.

The total number of consultations at Barbacena City Regional Hospital emergency care in 2019 for above 18 years of age patients equaled 24,809 . Thus $1.8 \%$ of hospital visits were motivated by headache complaints

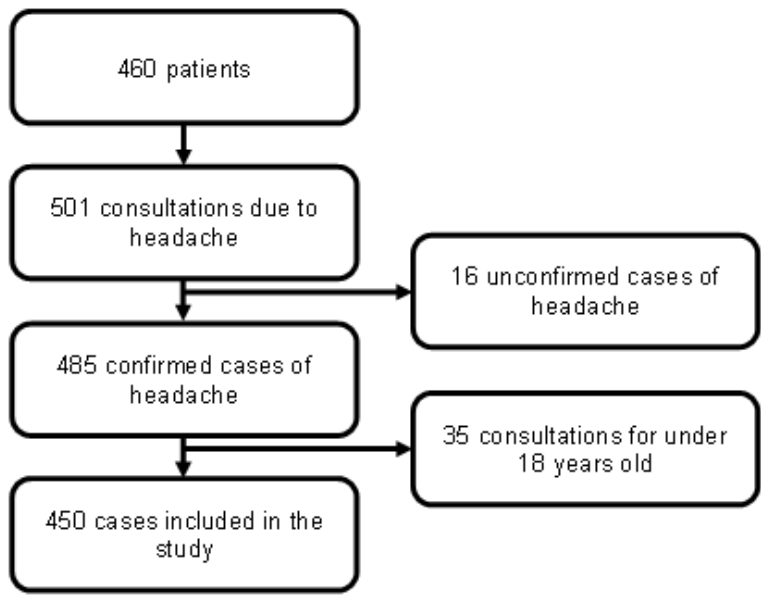

Figure 1. Selection of patients for the study.

Three hundred and twenty-one of the headache-motivated consultations corresponded to female patients (71\%) and 129 to male patients. Interconsultation with neurology services was necessary in 65 cases (14.4\%) and 69 patients (15.3\%) underwent head CT. Lumbar puncture realization for CSF analysis was needed in $11(2.4 \%)$ of events and a total of $12(2.6 \%)$ patients needed hospitalization (Table 1).

Table 1. Distribution of assistance according to variables of interest.

\begin{tabular}{c|c|c|c|}
\hline Age (years) & $41 \pm 15$ & n & $\%$ \\
\hline Gender & F & 322 & 71,6 \\
Interconsultation & No & 128 & 28,4 \\
with Neurologist & Yes & 385 & 85,6 \\
Head CT & No & 65 & 14,4 \\
CSF analysis & Yes & 381 & 84,7 \\
Hospitalization & No & 69 & 15,3 \\
& Yes & 439 & 97,6 \\
& No & 11 & 2,4 \\
\hline
\end{tabular}

CT: Computed Tomography; CSF: Cerebrospinal Fluid.

With respect to hospitalization time, 406 cases needed up to one day of observation (90.2\%) and the percentage of accumulated cases that demanded up to two days of hospitalization represented $95.3 \%$ of the sample. Sporadic cases demanded over five days of hospitalization.

Total expenditure (TE) was defined as the sum of imaging exams costs, medication costs, daily hospital stay costs, according to the following: 
TE: Imaging costs + Medication costs + (daily stay price + observation time).

According to documents obtained in the hospital's billing sector, daily stays in the emergency care were not charged. This caused these patients' expenditures to be much lower when compared to the rest of the consultations. To standardize costs calculation and minimize systematic errors in this variable with minimal loss, the formula mentioned above was deployed.

This resulted in estimated total expenditures with the headache patients care in 2019 equaling US\$90,855.60, with a median of US $\$ 129.73$ and an average of US $\$ 201.90$ per patient. Out of the total value, US\$1,827.09 (2.0\%) were spent on imaging tests and US\$4,735.21 (5.2\%) were spent on medication.

Among all 450 consultations, $25 \%$ presented imaging-related expenses, and $15.3 \%$ of the cases involved the performing of at least one head CT. In these cases, the imaging-related expenses median was US\$4.39.

Regarding the expenditures with medication, expenses reached the maximum values the equaled up to US $\$ 1,667.76$ per hospitalization. Only $1.49 \%$ of consultations did not compute onus.

Values of up to US $\$ 2.57$ represented an accumulated percentage of $90.0 \%$ of the consultations under study. Among the cases in which medication expenses were incurred, these presented a median of US\$0.52.

Most initial diagnoses were syndromic, and Headache (R51) corresponded to 299 (66.4\%) of consultations. Cases of Migraine (G34), Migraine without Aura (G430), Migraine with Aura (G431), and Other Migraine (G438) totalized 97 (21.6\%) of consultations and cases initially attributed to tension-type headache were the third most common motivation for seeking treatment - Table 2.

Table 2. Initial Diagnostics

\begin{tabular}{l|c|c}
\multicolumn{1}{c|}{ Initial Diagnostics } & $\mathbf{n}$ & $\%$ \\
R51 - Headache & 299 & 66,4 \\
Clustered cases of Migraine & 52 & 11,6 \\
G43 - Migraine & 20 & 4,4 \\
G430 - Migraine without aura [common migraine] & 17 & 3,8 \\
G431 - Migraine with aura [classic migraine] & 2 & 0,4 \\
G438 - Other forms of migraine & 6 & 1,3 \\
G439 - Migraine, unspecified & 5 & 1,1 \\
G44 - Other cephalic pain syndromes & 3 & 0,7 \\
G441 - Vascular headache, not elsewhere classified & 38 & 8,4 \\
G442 - Tension-type headache & 1 & 0,2 \\
G443 - Chronic post-traumatic headache & 2 & 0,4 \\
G444 - Drug-induced headache, not elsewhere classified & 5 & 1,1 \\
G448 - Other specified headache syndromes & 450 & 100,0
\end{tabular}

Thus, initial consultation, the number of cases with sufficient elements for classifying the headache as primary, according to the criteria established by the International ICHD- $3^{5}$, corresponded to 135 (30\%). As for the classification of the headache as secondary, this number equaled 6 cases (1.3\%). The remaining 309 cases (68.7\%) did not satisfy the criteria classification.

Regarding the final diagnoses registered in medical records, 224 cases $(49.8 \%)$ maintained the syndromic description of headache with no additional elements for etiological classification. Migraine variants were the principal etiological diagnostic in emergency care visits, accounting for a total of 102 (22.6\%) registered events. Tension-type headache was the second most prevalent etiology present in the discharge summaries, totalizing 26 cases (5.7\%). In 30 consultations, there was no register in the discharge summary clinical evolution in the patient's final evaluation.

Table 3. Final Diagnostics.

\begin{tabular}{|c|c|c|}
\hline Final Diagnostic & $\mathbf{n}$ & $\%$ \\
\hline Infectious and parasitic diseases (A00 - B99) & 2 & 0,4 \\
\hline Neoplasms (C00 - D48) & 1 & 0,2 \\
\hline Mental and behavioral disorders (F00 - F99) & 5 & 1,1 \\
\hline \multicolumn{3}{|l|}{ Nervous System Diseases (G00 - G99) } \\
\hline G00 - Bacterial meningitis & 1 & 0,2 \\
\hline G009 - Unspecified bacterial meningitis & 1 & 0,2 \\
\hline G039 - Unspecified meningitis & 1 & 0,2 \\
\hline G43 - Migraine & 52 & 11,6 \\
\hline G430 - Migraine without aura [common migraine] & 24 & 5,3 \\
\hline G431 - Migraine with aura [classic migraine] & 17 & 3,8 \\
\hline G438 - Other forms of migraine & 2 & 0,4 \\
\hline G439 - Migraine, unspecified & 5 & 1,1 \\
\hline G44 - Other cephalic pain syndromes & 4 & 0,9 \\
\hline G441 - Vascular headache, not elsewhere classified & 2 & 0,4 \\
\hline G442 - Tension-type headache & 26 & 5,8 \\
\hline $\begin{array}{l}\text { G444 - Drug-induced headache, not elsewhere } \\
\text { classified }\end{array}$ & 1 & 0,2 \\
\hline G448 - Other specified headache syndromes & 2 & 0,4 \\
\hline G510-Bell's Palsy & 2 & 0,4 \\
\hline Circulatory System Diseases (100 - 199) & 8 & 1,8 \\
\hline Respiratory System Diseases (J00 - J99) & 4 & 0,9 \\
\hline Musculoskeletal System Diseases (M00 - M99) & 5 & 1,1 \\
\hline Genitourinary System Diseases (N00 - N99) & 2 & 0,4 \\
\hline $\begin{array}{l}\text { Abnormal symptoms and signs from clinical and laboratory } \\
\text { examinations, not elsewhere classified (R00 - R99) }\end{array}$ & 6 & 1,2 \\
\hline R51 - Headache & 224 & 49,8 \\
\hline R51 - Headache + another ICD & 19 & 4,2 \\
\hline External causes injuries (S00 - T98) & 4 & 0,9 \\
\hline No discharge summary & 30 & 6,7 \\
\hline Total & 450 & 100 \\
\hline
\end{tabular}


Table 3 shows the final diagnoses organized according to disease group, following the ICD-10.10 Diagnoses under the "nervous system diseases" group were differentiated according to the specific ICD. In the case of the "Symptoms, signs and abnormal clinical and laboratory findings, not elsewhere classified", it became evident that the headache ICD (R51) was employed in isolation or in association to other codes.

After the individual medical records analysis, a reclassification of primary and secondary headaches was performed following the criteria established by ICHD-3. ${ }^{5}$ Primary headache preserved a similar proportion in final diagnoses in relation to initial diagnoses (38.9\% and $30 \%$ respectively). Secondary headaches presented a significant increase in the final evaluations $(31.1 \%$ versus $1.3 \%$ in initial evaluations). For a significant portion of patients, data described in the medical records were still insufficient for such classification (135 cases, which corresponds to $30 \%$ of consultations), although in a lower proportion in comparison to the initial consultation (68.7\%) - Figure 2.

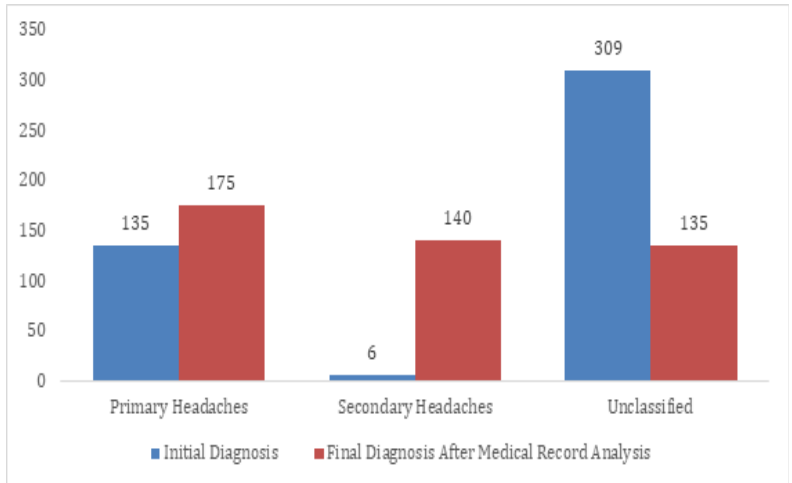

Figure 2. Comparison of initial and final diagnoses after analysis of medical records regarding primary and secondary headaches.

Out of the 315 consultations in which the distinction between primary and secondary headaches was achievable, interconsultation with neurology services was requested in 23 primary headache cases and in 38 secondary headache cases. Chances of interconsultation with neurology were 2.46 times higher $(95 \% \mathrm{Cl} 1.38$ to 4.38$)$ for secondary headache than in primary headaches $\left(X^{2} p=0.002\right)$. Chances of head CT were 4.55 times higher for secondary headache cases $\left(95 \% \mathrm{Cl} 2.44\right.$ to $\left.8.52-X^{2} p<0.001\right)$. CSF analysis was conducted in only one primary headache case compared to 9 punctures requested for secondary headaches. Only one primary headache case resulted in hospitalization - Table 4.
Table 4. Proportion of cases of primary and secondary headache according to variables of interest.

\begin{tabular}{|c|c|c|c|c|c|}
\hline & \multicolumn{2}{|c|}{$\begin{array}{l}\text { Secondary } \\
\text { Headache }\end{array}$} & $\begin{array}{l}\text { Primary } \\
\text { Headache }\end{array}$ & $\begin{array}{l}\text { Odds } \\
\text { Ratio }^{\circ}\end{array}$ & $\mathbf{p}^{*}$ \\
\hline \multirow{2}{*}{$\begin{array}{l}\text { Interconsultation } \\
\text { with Neurologist }\end{array}$} & Yes & 38 & 23 & \multirow{2}{*}{2,46} & \multirow{2}{*}{0,002} \\
\hline & No & 102 & 152 & & \\
\hline \multirow{2}{*}{ Head CT } & Yes & 44 & 16 & \multirow{2}{*}{4,55} & \multirow{2}{*}{$<0,001$} \\
\hline & No & 96 & 159 & & \\
\hline \multirow{2}{*}{ CSF analysis } & Yes & 9 & 1 & \multirow{2}{*}{ 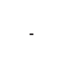 } & \multirow[t]{2}{*}{$0,006^{b}$} \\
\hline & No & 131 & 174 & & \\
\hline \multirow{2}{*}{ Hospitalization } & Yes & 11 & 1 & \multirow{2}{*}{ 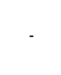 } & \multirow[t]{2}{*}{$0,002^{b}$} \\
\hline & No & 129 & 174 & & \\
\hline
\end{tabular}

$N=315$. a Secondary / Primary. ${ }^{*}$ Chi- square $\left(x^{2}\right)$ unless otherwise indicated. $b$ Fischer's exact.

CT: Computed Tomography; CSF: Cerebrospinal Fluid.

The median age was higher for the secondary headache group (41 years of age, compared to 38 for primary), presenting a significantly distinct distribution ( $p=0.019$, Mann-Whitney test). Similarly, higher costs related to imaging were attributed to secondary headache, with values reaching up to US\$336.92 (average US\$10.35 \pm 3.19 ), in comparison to a maximum cost of US\$18.54 (average US\$1.01 \pm 0.24 ) for primary headaches, with significantly different cost distribution curves $(p<0.001$, MannWhitney test). However, there were no significant differences in medication costs, hospital stay, or total costs for the two headache groups (Mann-Whitney test, 0.951, p = 0.229 and 0.275 respectively) - Table 5 .

Table 5. Age and costs according to secondary and primary headache.

\begin{tabular}{|c|c|c|c|c|c|c|c|}
\hline Variable & & $\begin{array}{l}\text { Primary } \\
\text { Heada- } \\
\text { che }\end{array}$ & $\begin{array}{l}\text { Secun- } \\
\text { dary } \\
\text { Heada- } \\
\text { che }\end{array}$ & $p_{0}$ & $\begin{array}{l}\text { Hospitali- } \\
\text { zation }\end{array}$ & $\begin{array}{l}\text { Non- } \\
\text { hospi- } \\
\text { taliza- } \\
\text { tion }\end{array}$ & $\mathrm{pb}$ \\
\hline \multirow{3}{*}{$\begin{array}{c}\text { Age } \\
\text { (years) }\end{array}$} & Median & 38 & 41 & & 57 & 40 & \multirow{3}{*}{0,043} \\
\hline & Minimum & 18 & 18 & 0,019 & 18 & 18 & \\
\hline & Maximum & 85 & 89 & & 89 & 92 & \\
\hline \multirow{3}{*}{$\begin{array}{l}\text { Costs } \\
\text { related to } \\
\text { imaging } \\
\text { test } \\
\text { (US \$) }\end{array}$} & Median & 0,00 & 0,00 & & 23,80 & 0,00 & \multirow{3}{*}{$<0,001$} \\
\hline & Minimum & 0,00 & 0,00 & $<0,001$ & 0,00 & 0,00 & \\
\hline & Maximum & 18,54 & 336,92 & & 336,92 & 126,25 & \\
\hline \multirow{3}{*}{$\begin{array}{c}\text { Medication } \\
\text { costs } \\
\text { (US \$) }\end{array}$} & Median & 0,56 & 0,50 & & 74,04 & 0,51 & \multirow{3}{*}{$<0,001$} \\
\hline & Minimum & 0,00 & 0,00 & 0,951 & 2,57 & 0,00 & \\
\hline & Maximum & 155,32 & $1.667,76$ & & $1.667,76$ & 155,54 & \\
\hline \multirow{3}{*}{$\begin{array}{l}\text { Hospital } \\
\text { stay costs } \\
\text { (US \$) }\end{array}$} & Median & 135,00 & 129,48 & & $1.362,58$ & 123,95 & \multirow{3}{*}{$<0,001$} \\
\hline & Minimum & 88,51 & 88,51 & 0,229 & 311,47 & 88,51 & \\
\hline & Maximum & $1.639,75$ & $5.949,70$ & & $5.949,70$ & 712,47 & \\
\hline \multirow{3}{*}{$\begin{array}{l}\text { Total costs } \\
\text { (US \$) }\end{array}$} & Median & 136,14 & 135,24 & & $1.438,57$ & 127,26 & \multirow{3}{*}{$<0,001$} \\
\hline & Minimum & 88,99 & 88,51 & 0,275 & 332,58 & 88,51 & \\
\hline & Maximum & $1.644,23$ & $6.606,18$ & & $6.606,18$ & 736,63 & \\
\hline
\end{tabular}

a Mann Whitney U Test, $\mathrm{n}=315$. b Mann Whitney U Test, $\mathrm{n}=450$. 
Total costs were higher among the group of patients who needed hospitalization regardless of headache classification, with a median of US\$1,483.57. For patients who did not require hospitalization, the median of total costs equaled US\$127.26, with a significantly different distribution in comparison to those in in-patient care $(\mathrm{p}<0.001$, Mann-Whitney test). The median age equaled 57 years of age for in-patients (IQR 37) compared to 40 years of age for patients who were not hospitalized (IQR 23), presenting a significantly different distribution - Table 5 .

Total costs did not vary significantly according to $\operatorname{sex}(p=0.495$, Mann-Whitney test). The same was observed for age and the other cost categories ( $p>0.05$, Mann-Whitney test).

\section{Discussion}

Headache is one of the most frequent conditions reported in medical practice. ${ }^{1,11,12}$ It causes incapacitation and loss of life, motivating a great part of patients suffering from this symptom to search for urgency and emergency care. According to some studies, headaches respond to $0.5 \%$ to $2.8 \%$ of consultations in emergency care units. ${ }^{13,14}$ Our study endorses these statistics, showing that headache complaints corresponded to $1.8 \%$ of consultations in the emergency care department of the Barbacena City's Regional Hospital in 2019.

Headaches etiological diagnosis was proven to be a challenge, as described in previous accounts. $2,4,1530 \%$ of patients were discharged with no definitive diagnosis of the cause for their symptoms, as observed in another study. ${ }^{16}$ The final IDC-10 attributed to $49.8 \%$ of the cases was solely Headache (R51). In other words, almost half of the patients left the hospital having received no explanation for their symptoms. It is known that efficient headache treatment is directly connected to the diagnosis according to the ICHD-3 criteria $^{5,15}$, which cannot be determined for an expressive portion of patients evaluated in our study.

Among the available methods for determining headache causes, interconsultations with specialists were 2.46 times more used in secondary headache cases than in primary headache cases. Head CTs were performed 4.55 more times for secondary headaches; CSF analysis and necessity of hospitalization also occurred in a higher proportion than secondary headaches when compared to primary headaches. The study identified significant differences for all parameters. Thus, imaging test costs were more prominent for the secondary headache group (Average US $\$ 10.35 \pm 3.19$ ) than for the primary headache group (Average US $\$ 1.01 \pm 0.24$ ). However, no significant differences in medication costs, hospital stay costs, and total cost of consultation among the two headache groups.

The estimated expenditures with headache patients in Barbacena
City Regional Hospital equated US\$90,855.60 (average US $\$ 201.90$ per patient) in 2019. This confirms the great financial impact generated by this condition. 79,12

Part of headache patient-related expenditures included the performance of imagining tests. In this study, head CT costs corresponded to $2 \%$ of the total value. Nonetheless, it has been demonstrated that out of all head CTs performed in headache or facial patients at urgency departments, $95 \%$ may be normal. ${ }^{4,14}$ Therefore, identifying which cases actually require imaging tests can be an important factor in expenditure control.

As a transversal retrospective cohort study based on medical records analysis, this study presented some limitations. Since patient follow-up after discharge is not available, the diagnostic confirmation for suspected conditions reported in the medical records could not be confirmed. Diagnoses were determined based on the emergency care records, which may not have been precise. Oftentimes, specific and important features of a patient's history and physical exams were not available and, thus, the etiology attributed to headache could not be accurately analyzed. Additionally, the definition of hospital expenditures was based on the Brazilian National Health System's table, with standardized values in which costs related to procedures performed during hospital care are based.

However, these values represent only a portion of what is actually spent in practice, since costs related to physical structure maintenance, CT equipment maintenance, human resources necessary for patient care, supplies used in laboratory exams, among other costs, were not included in the estimations. Thus, we can draw only limited conclusions about the rational use of available resources for the diagnosis of headaches and the actual expenditures related to patient care.

On the other hand, the strength of this study is allowing the demonstration of the expressive financial impact patients with headache complaints entail to the hospital, even taking into consideration that the displayed values correspond only to a portion of actual expenditures. Therefore, this study emphasizes the importance of accurate diagnosis and rational use of available resources for better management of these patients.

\section{Conclusion}

The socio-economic impactcaused by headaches is unquestionable. It is a highly frequent symptom and both its etiological distinction and adequate treatment require solid evaluation. Due to the resources spent in its evaluation and monitoring, headaches can be considered a public health problem. Therefore, this study suggests that resources should be allocated in the health education and professional training for the proper conduction of these 
patients, so that they may benefit from an optimized treatment of their condition without overwhelming the health system.

\section{Ethic Approval and Patient Consent}

All procedures in this study were in accordance with the ethical standards of the responsible committee on human experimentation from Hospital Foundation of the State of Minas Gerais - FHEMIG with approval number 4.127.307. Informed consent was obtained from all patients for being included in the study.

Conflict of interest: None

Financing: None

Lígia Barros de Oliveira: Conceptualization, Data curation, Investigation, Project administration, Resources, Visualization, Writing - original draft.

Jessica Guimarães: Data curation, Investigation, Resources, Writing - original draft.

Danilo Jorge da Silva: Data curation, Formal analysis, Software, Writing - original draft.

Mauro Eduardo Jurno: Conceptualization, Investigation, Methodology, Project administration, Resources, Supervision, Visualization, Writing - review \& editing

Lígia Barros de Oliveira

https://orcid.org/ 0000-0002-6715-6590

Jessica Guimarães

https://orcid.org/ 0000-0003-0640-0470

Danilo Jorge da Silva

https://orcid.org/ 0000-0002-8086-1200

Mauro Eduardo Jurno

https://orcid.org/ 0000-0002-8743-9395

\section{References}

1. Queiroz LP and Silva Junior AA. The prevalence and impact of headache in Brazil. Headache 2015;55 Suppl 1:32-38 Doi: 10.1111/head. 12511

2. Nye BL and Ward TN. Clinic and Emergency Room Evaluation and Testing of Headache. Headache 2015;55(9):1301-1308 Doi: $10.1111 /$ head. 12648

3. Munoz-Ceron J, Marin-Careaga V, Peña L, Mutis J and Ortiz $G$. Headache at the emergency room: Etiologies, diagnostic usefulness of the ICHD 3 criteria, red and green flags. J PLOS One 2019; 14(1):e0208728
4. Minen MT, Tanev K and Friedman BW. Evaluation and treatment of migraine in the emergency department: a review. Headache 2014;54(7): $1131-1145$ Doi: 10.1111/head. 12399

5. Headache Classification Committee of the International Headache Society (IHS) The International Classification of Headache Disorders, 3rd edition. Cephalalgia 2018;38(1):1-211 Doi: 10.1177/0333102417738202

6. Speciali JG, Kowacs F, Jurno ME, Bruscky IS, de Carvalho JJF, Malheiro FG, . . . Pires 10 DBFJABdN. Protocolo nacional para diagnóstico e manejo das cefaleias nas unidades de urgência do Brasil - 2018. Ac Bras Neurologia 2018

7. Bigal ME, Rapoport AM, Bordini CA, Tepper SJ, Sheftell FD and Speciali JG. Burden of migraine in Brazil: estimate of cost of migraine to the public health system and an analytical study of the cost-effectiveness of a stratified model of care. Headache 2003;43(7):742-754 Doi: 10.1046/i.1526-4610.2003.03132.x

8. Oliveira $A B$, Queiroz LP, Sampaio Rocha-Filho P, Sarmento EM and Peres MF. Annual indirect costs secondary to headache disability in Brazil. Cephalalgia 2020;40(6):597-605 Doi: 10.1177/0333102419889357

9. Negro A, Sciattella P, Rossi D, Guglielmetti M, Martelletti P and Mennini FS. Cost of chronic and episodic migraine patients in continuous treatment for two years in a tertiary level headache Centre. J Headache Pain 2019;20(1):120 Doi: 10.1186/ s10194-019-1068-y

10. DiSantostefano J. International Classification of Diseases 10th Revision (ICD-10). The Journal for Nurse Practitioners 2009;5(1):5657 Doi: 10.1016/i.nurpra.2008.09.020

11. Bigal ME, Bordini CA and Speciali JG. Tratamento da cefaléia em uma unidade de emergência da cidade de Ribeirão Preto. Arq. Neuro-Psiquiatr 1999;57:813-819

12. Bigal ME, Bordini CA, Speciali JJ and Pain F. Etiology and distribution of headaches in two Brazilian primary care units. Headache 2000;40(3):241-247

13. Munoz-Ceron J, Marin-Careaga V, Peña L, Mutis J and Ortiz $G$. Headache at the emergency room: Etiologies, diagnostic usefulness of the ICHD 3 criteria, red and green flags. PLOS One 2019; 14(1):e0208728 Doi: 10.1371/journal.pone.0208728

14. Goldstein JN, Camargo CA, Jr., Pelletier AJ and Edlow JA. Headache in United States emergency departments: demographics, work-up and frequency of pathological diagnoses. Cephalalgia 2006;26(6):684-690 Doi: 10.1111/j.1468-2982.2006.01093.x

15. O'Flynn $N$ and Ridsdale L. Headache in primary care: how important is diagnosis to management? $\mathrm{Br} J$ Gen Pract 2002;52(480):569-573

16. Moeller JJ, Kurniawan J, Gubitz GJ, Ross JA and Bhan V. Diagnostic accuracy of neurological problems in the emergency department. Can J Neurol Sci 2008;35(3):335-341 Doi: 10.1017/ s0317167100008921 